\title{
Proposition d'une approche de reconfiguration des processus métiers de la chaîne logistique
}

\author{
An approach for business processes reconfiguration
}

\author{
Slim Zidi ${ }^{1}$, Nadia Hamani ${ }^{2}$, Lyes Kermad ${ }^{1}$ \\ ${ }^{1}$ Laboratoire QUARTZ (EA 7393), Université de Paris 8, 2 rue de la Liberté - 93526 Saint-Denis cedex \\ slim.zidi02@etud.univ-paris8.fr, I.kermad@iut.univ-paris8.fr \\ ${ }^{2}$ Laboratoire des Technologies Innovantes, 48 rue d'Ostende 02100 Saint-Quentin cedex, nadia.hamani@u-picardie.fr
}

\begin{abstract}
RÉSUMÉ. La recherche perpétuelle de flexibilité conduit les entreprises à mettre en place des chaînes logistiques reconfigurables. Dans cet article, nous nous intéressons à la reconfiguration des chaînes logistiques à travers les processus métiers qui les composent. Nous proposons une approche de reconfiguration basée sur un modèle intégré QFD (Quality Function Deployment)/AHP (Analytic Hierarchy Process) qui permet de déterminer les processus, les activités, les rôles et les acteurs qui nécessitent des reconfigurations. Cette approche a pour but de déterminer le scénario de reconfiguration le plus approprié ainsi que le processus qui doit être reconfiguré en priorité.

ABSTRACT. The continuous search for flexibility leads companies to implement reconfigurable supply chains. In this article, we focus on the reconfiguration of supply chains through the business processes that compose them. We propose an approach of reconfiguration based on an integrated QFD (Quality Function Deployment)/AHP (Analytic Hierarchy Process) model that allows to determine the processes, activities, roles and actors that require reconfigurations. Our aims is to determine the appropriate reconfiguration scenario and the process that should be reconfigured as a priority.

MOTS-CLÉS. Reconfiguration, Processus métiers, Chaîne logistique, Déploiement de la Fonction Qualité (DFQ), Analyse Hiérarchique des Procédés (AHP).

KEYWORDS. Reconfiguration, Business process, Supply chain, Quality Function Deployment (QFD), Analytic Hierarchy Process (AHP).
\end{abstract}

\section{Introduction}

De nos jours, les entreprises sont soumises à une forte concurrence. Face à un marché qui est devenu de plus en plus changeant et incertain, l'objectif est de pouvoir modifier la configuration de la chaine logistique avec les moindres ressources. Etant donné que le problème de reconfiguration des systèmes de production et des chaînes logistiques est assez récent, nous nous intéressons aux travaux élaborés depuis l'apparition du concept de la reconfiguration des systèmes de production par Koren et al. (1999). Notre recherche nous a permis de trouver plusieurs travaux dans cette thématique. Néanmoins, nous nous focalisons sur les travaux qui ont proposé des modèles, des approches et des méthodes pour la conception des chaînes logistiques reconfigurables.

Selon Chandra et al. (2016), une chaîne reconfigurable est une chaîne flexible et apte à modifier sa configuration en optimisant ses ressources et sans perdre son efficacité opérationnelle, tout en répondant à la modification des exigences clientèles ou de l'environnement opérationnel.

Plusieurs auteurs ont traité la reconfiguration afin de mettre en place des chaînes et réseaux logistiques qui sont capables de s'adapter aux changements (Thierry et al. (2008); Ivanov et al. (2009)). La reconfiguration des chaînes logistiques passe essentiellement par leurs systèmes de production, leurs systèmes d'information et leurs processus métiers. En effet, ces derniers jouent un rôle crucial dans l'assurance d'une chaîne reconfigurable. Plusieurs approches ont été proposées pour répondre à cette problématique.

Les processus métiers sont définis comme étant un ensemble de procédures et d'activités qui sont liés entre eux, qui permettent d'organiser le travail entre les rôles confiés aux différents acteurs Morley et al. (2011). En effet, le processus métier est un ensemble de tâches ordonnées qui permet de convertir 
les entrées en sorties pour atteindre un objectif fixé auparavant. Plusieurs approches ont été proposées dans le cadre de la reconfiguration des processus métiers de la châne logistique. Notre objectif est de proposer une approche de reconfiguration en étendant les travaux de Kermad (2018) au niveau des processus métiers de la chaîne logistique. Ce dernier a proposé une approche de conduite du changement établie pour la mise en place d'un ERP (Entreprise Resource Planning). Par ailleurs, nous allons nous baser sur les concepts relatifs aux processus métiers définis par Morley et al. (2011) pour pouvoir identifier le scénario de reconfiguration qui permet de réussir la reconfiguration du processus.

Le reste de l'article est organisé comme suit. Dans la section 2, nous présentons un état de l'art sur les modèles proposés pour la reconfiguration des chaînes logistiques. Ensuite, nous étudions en particulier la reconfiguration des chaînes logistiques à travers les processus métiers pour déterminer les concepts traités (activité, rôle, ressource...). Ensuite, nous proposons une approche de reconfiguration des processus métiers de la chaîne logistique. L'approche proposée est une hybridation QFD (Quality Function Deployment)/AHP (Analytic Hierarchy Process) qui a pour but de déterminer le scénario de reconfiguration le plus approprié ainsi que le processus qui doit être reconfiguré en priorité.

\section{Revue de la littérature}

\subsection{Modélisation des chaînes logistiques reconfigurables}

Il existe plusieurs méthodes et outils pour concevoir des modèles de chaînes logistiques reconfigurables. Selon Chandra et al. (2016), les modèles de la chaîne logistique peuvent-être divisés en cinq types :

- Les modèles conceptuels : qui définissent les problèmes de configuration et d'information de la chaîne logistique. Ce type de modélisation concerne aussi la reconfiguration des processus métiers ;

- Les modèles analytiques : qui incluent les modèles de programmation mathématique qui peuvent être déterministes ou stochastiques ;

- Les modèles de simulation : qui décrivent l'aspect dynamique de la configuration/reconfiguration des chaînes logistiques ;

- Les modèles hybrides: l'hybridation des différents types de modèles de configuration/reconfiguration des chaînes logistiques qui ne se limite pas seulement à l'hybridation des modèles de simulation et analytiques ;

- Les modèles statistiques : se basent sur la description des configurations des chaînes logistiques à base d'historiques de données, qui peuvent fournir des fonctions supports pour la configuration.

\subsection{Les approches de reconfiguration}

\subsubsection{La reconfiguration des chaînes logistiques}

Ross et al. (1998) présentent une méthodologie de reconfiguration d'un réseau de la chaîne logistique qui se caractérise par deux niveaux :

- Le premier niveau s'intéresse à l'évaluation de la performance du réseau et l'identification des actions efficaces ;

- Le deuxième niveau s'intéresse à l'élaboration d'un modèle qui intègre les actions efficaces afin de reconfigurer le réseau.

Cette méthodologie permet de fournir un cadre d'aide à la décision pour tous les processus. Une étude de cas a été réalisée dans cet article dans le cadre de la reconfiguration d'un réseau logistique dans le secteur pétrolier, dont les auteurs se sont appuyés sur la méthode DEA (Data Envelopment Analysis) développé par Charnes et al. (1978) pour estimer l'efficience des opérations, et sur la programmation en nombres entiers afin de déterminer et de mesurer l'efficacité des différentes actions ou opérations choisies. 
Dans le cadre de l'amélioration des processus et leurs interactions, Chandra et al. (2002) proposent une approche qui consiste à concevoir et développer des configurations pour la chaîne logistique. Cette approche est basée sur « le modèle processus » qui permet de définir les structures organisationnelles, les interactions, les configurations et les orientations de production dans la chaîne logistique. Le modèle proposé est basé sur ARIS (Architecture of Integrated Information Systems) pour faire la modélisation des processus, et sur SAP (Systems, Applications and Products) pour la base de données des processus. Chandra et al. (2004) traitent les problèmes de la reconfiguration dans les chaînes logistiques à partir de la modélisation des entreprises dans leurs niveaux stratégiques et décisionnels. En effet, ils ont conçu un système d'aide à la décision DSS (Decision Support System) qui sert à développer des configurations pour les chaînes logistiques en associant des scénarios et en mettant en œuvre plusieurs stratégies et politiques. Ce système est composé d'un DMS (Decision Modeling System) qui permet de résoudre les problèmes liés aux chaînes logistiques, et d'un ISS (Information Support System) qui est un sous-système permettant de fournir un support d'information pour la modélisation et les informations liées à la conception du produit et aux exigences des clients.

Dans l'optique de l'amélioration du DMS, Chandra et al. (2004) proposent un modèle composé de 2 parties. Un modèle d'optimisation qui a pour but d'établir la configuration des chaînes logistiques, et un modèle de simulation qui permet d'évaluer les configurations établies. La démarche proposée consiste en :

- L'élaboration d'un modèle de processus et pour sa base de données pour avoir une représentation complète sur tous les problèmes de la chaîne logistique ;

- La réalisation des tentatives de reconfiguration. Ce sont des alternatives pour la configuration initiale qui vont être stockées dans la base de données déjà élaborée dans la première étape ;

- L'élaboration d'un modèle de décision qui permet de faire des évaluations sur ces tentatives selon plusieurs scénarios ;

- L'élaboration d'un modèle d'aide à la décision et accumulation des connaissances.

Par ailleurs, Chandra et al. (2016) décrivent un modèle générique qui met l'accent sur les problèmes de configurations des chaînes logistiques. Plusieurs modèles ont été proposés :

- Goal model (incluant 3 objectifs) : stratégique, structurel et de performance.

- Actor model (incluant 3 types d'entités) : partenaires, gestionnaires et clients.

- Process model (modèle SCOR (Supply Chain Operations Reference)): planification, approvisionnement, production, distribution et retour.

Ainsi, la méthodologie de reconfiguration des chaînes logistiques proposée consiste à :

- Définir les capacités de prise de décision pour chaque unité de la chaîne logistique en s'appuyant sur les stratégies de la chaîne et sur la base des données et des connaissances ;

- Déterminer les problèmes de configuration de la chaîne logistique ;

- Définir les problèmes de prise de décision des configurations de la chaîne logistique.

Dans le cadre des réseaux collaboratifs ayant une structure dynamique, Ivanov et al. (2009) ; Ivanov et al. (2010) proposent des algorithmes pour assurer la reconfiguration de ces réseaux basés sur «la théorie du contrôle du réseau collaboratif ». Ces modèles permettent d'intégrer la reconfiguration dans la boucle de contrôle du réseau collaboratif et d'interconnecter les phases de planification et d'exécution pour augmenter l'efficacité du réseau collaboratif. 
Pour la planification multi-structurelle et les opérations des chaînes logistiques adaptables aux structures dynamiques, Ivanov et al. (2010) proposent une approche basée sur l'application combinée de la théorie du contrôle, la recherche opérationnelle et la modélisation basée sur les agents. Le modèle se compose de cinq blocs :

- supply chain (bloc $n^{\circ} 1$ ), planning bloc (bloc ${ }^{\circ} 2$ ) et measuring bloc (bloc ${ }^{\circ} 3$ ) qui assurent le fonctionnement de la chaîne logistique et minimisent les écarts entre les exigences et les outputs ;

- Input vector (bloc $\left.\mathrm{n}^{\circ} 4\right)$ et control bloc (bloc $\mathrm{n}^{\circ}$ ) assurent le suivi et la correction des actions en cas de perturbation.

Pour la reconfiguration des chaînes logistiques dans un environnement stochastique, Dev et al. (2014) traitent l'identification des outils opérationnels en s'appuyant sur la méthode ISM (Interpretive Structural Modeling) qui représente un modèle hiérarchique. Ce modèle permet de déterminer les interactions entre les différents facteurs de la chaîne logistique après avoir analysé les mesures de leurs performances. Afin d'adapter et comprendre les facteurs clés permettant d'assurer la performance des chaînes logistiques, Dev et al. (2016) proposent une intégration entre la simulation multi-agents et l'arbre de décision afin d'estimer la performance des niveaux moyens d'inventaire. La reconfiguration des chaînes logistiques permet d'améliorer la performance au niveau des règles de décision.

Afin de réussir la reconfiguration des chaînes logistiques, plusieurs auteurs ont mis le point sur la reconfiguration des processus métiers de la chaîne logistique qui permet d'adapter les processus avec le système d'information.

\subsubsection{La reconfiguration des processus métiers}

Morley et al. (2011) considèrent que l'amélioration s'effectue par la reconfiguration des processus métiers et les processus systèmes d'information d'une part, et par la maturité des processus d'autre part. La reconfiguration vise à atteindre la résilience, l'efficience, la flexibilité et l'amélioration de la relation client. Plusieurs approches ont été proposées dans le cadre de la reconfiguration des processus métiers de la chaîne logistiques. Partant des modèles qui ont pris en compte la phase de changement ou de reconfiguration dans les processus métiers, nous avons cherché dans ces travaux à définir les différents concepts de reconfiguration qui ont été intégrés lors de la conception et de la modélisation des processus métiers.

Mashari et al. (2000) traitent le problème de l'implémentation de l'ERP dans l'optique de reconfigurer la chaîne logistique du point de vue organisationnel et structurel. Dans cet article, les auteurs pointent le rôle que joue particulièrement SAP dans la création de la valeur dans les chaînes logistiques afin de garantir une chaîne logistique intégrée et d'améliorer les interactions dans le réseau des processus métiers.

Trienekens et al. (2001) proposent un ensemble de méthodes de modélisation pour la reconfiguration des chaînes logistiques. Chaque modèle présente une dimension particulière de la gestion de la chaîne logistique. Cet article est inspiré des méthodologies proposées par Vernadat (1992) pour analyser et reconfigurer les processus métiers. Pour ce faire, les auteurs se sont basés sur 3 types de modélisation : EPC (Event Process Chain), ACM (Activity Chain Model) et la méthode GRAI (Grid Method) ajustée aux chaînes logistiques. Ainsi, la première modélisation se focalise sur les relations temporelles entre les processus/les fonctions et les orientations clients qui permettent d'optimiser les ressources. La deuxième modélisation porte sur les fonctions/processus et les flux d'informations qui permettent de réduire le temps et améliorer les processus. La troisième modélisation concerne les structures de décision et les flux d'informations qui permettent d'améliorer les structures décisionnelles et les flux d'informations.

Changchien et al. (2002) abordent le problème de reconfiguration des processus métiers au niveau des chaînes logistiques afin d'assurer l'amélioration de la performance de ces deniers. Ils proposent 
une méthodologie basée sur un ensemble d'étapes en utilisant une matrice d'analyse et une simulation orientée objet. En effet, la première étape consiste en la création d'une vision et des objectifs c'est-àdire l'entreprise devrait revoir son actuel profil et développer une vision stratégique globale. Les activités clés de cette étape sont de faire une évaluation de la structure organisationnelle et de l'environnement en identifiant les besoins et en fixant des objectifs de reconfiguration. La deuxième étape consiste à identifier le processus à reconfigurer étant donné que les processus représentent les éléments d'une entreprise.

Kelepouris et al. (2006) présentent une conception d'un réseau de chaîne logistique intelligent à travers la mise en œuvre d'un réseau logistique reconfigurable pour répondre aux différentes exigences. Ils ont développé un modèle de réseau de chaîne logistique reconfigurable à travers les techniques de modélisation des entreprises et des réseaux de chaîne en se basant sur les modèles des systèmes de production reconfigurables. Afin de construire un réseau reconfigurable, les auteurs ont mentionné que les entreprises doivent concevoir leur réseau des chaînes logistiques en utilisant des règles de conception visibles.

Masood et al. (2013) proposent une approche basée sur des modèles pour que les processus métiers et les systèmes de production puissent être reconfigurables et transformables. Pour ce faire, ils se sont basés sur le concept de rôle d'une part, pour répondre à la question « qui » et le concept de ressource d'autre part, pour répondre à la question « comment» de telle sorte que le décideur, c'est-à-dire celui qui prend en charge la décision de reconfiguration, puisse faire face aux exigences du changement.

Palma-Mendoza et al. (2014) traitent le concept de la reconfiguration des processus métiers dans le cadre de la construction des chaînes logistiques. La démarche proposée se base sur l'analyse SWOT (Strengths, Weaknesses, Opportunities, Threats) pour identifier les activités relatives aux processus métiers qui vont subir les actions de reconfiguration. Le modèle SCOR permet de décrire et évaluer les chaînes logistiques. Ensuite, le choix des processus et des activités à reconfigurer es basé sur le la méthode AHP. Finalement, afin d'implémenter les changements, les auteurs ont proposé de faire une analyse des processus AS-IS et une conception des processus AS-TO-BE.

\begin{tabular}{|c|c|c|}
\hline Les articles & Méthode appliquée & $\begin{array}{c}\text { Niveau de } \\
\text { reconfiguration }\end{array}$ \\
\hline Mashari and Zairi (2000) & SAP & Processus, activités \\
\hline $\begin{array}{l}\text { Trienekens and Hvolby } \\
\text { (2001) }\end{array}$ & EPC, ACM, GRAI & $\begin{array}{l}\text { Processus, activités, } \\
\text { ressources }\end{array}$ \\
\hline $\begin{array}{l}\text { Changchien and Shen } \\
\text { (2002) }\end{array}$ & $\begin{array}{c}\text { Core Process Analysis } \\
\text { Matrix, Object Oriented } \\
\text { Simulation }\end{array}$ & Processus \\
\hline Kelepouris et al. (2006) & $\begin{array}{c}\text { Modèle } \\
\text { d'Entreprise/Modèle SCOR }\end{array}$ & $\begin{array}{c}\text { Processus, activités, } \\
\text { ressources }\end{array}$ \\
\hline Masood et al. (2013) & $\begin{array}{c}\text { CIMOSA (Computer } \\
\text { Integrated Manufacturing } \\
\text { Open Systems } \\
\text { Architecture) }\end{array}$ & Processus, ressources, rôles \\
\hline Palma-Mendoza et al. & AHP, Modèle SCOR & Processus, activités \\
\hline
\end{tabular}

Tableau 1. Classification des articles selon le niveau de reconfiguration

Ces articles traitent la reconfiguration des chaînes logistiques à travers leurs activités, ressources et les rôles associés aux processus métiers comme l'illustre le tableau 1. Notre objectif est d'aborder la reconfiguration des chaînes logistiques à travers leurs processus métiers, en particulier à travers leurs activités, rôles et acteurs afin d'assurer des actions de reconfiguration sur les processus qui nécessitent ces actions en priorité. La plupart des changements proposés auparavant ont été appliqués dans le cadre de l'informatisation des processus ou des activités qui posent le plus de problèmes. En outre, ce changement traite les étapes relatives à l'implantation d'un système d'information au sein d'une 
entreprise. Cependant, les travaux qui ont traité la phase de changement suite à un aléa ou un dysfonctionnement qui peut affecter les processus métiers d'une chaîne logistique sont peu développés.

\section{Proposition d'une approche de reconfiguration}

Notre travail part de la définition des objectifs de la reconfiguration à la suite d'un évènement déclencheur qui induit le besoin de la reconfiguration. Par la suite, afin de faire face à ces perturbations, plusieurs scénarios sont mis à la disposition du décideur. Le choix des scénarios doit être fait d'une manière objective, c'est-à-dire que le décideur fixe un ensemble de critères dans le but de choisir le scénario le plus approprié pour les processus métiers de la chaîne logistique. L'étape suivante porte sur l'analyse des processus. En effet, nous allons aborder cette phase par l'identification des ensembles de processus métiers pour pouvoir dégager leur corrélation et les scénarios de reconfiguration déterminés auparavant.

Deuxièmement, l'identification des activités associées à chaque processus et la détermination de la corrélation entre ces activités. Cette étape permet d'identifier les différents rôles de chaque activité.

Ensuite, il faut définir les acteurs à qui ont été confiés les différents rôles identifiés préalablement et déterminer les interactions entre ces acteurs. L'objectif de cette étape est de prioriser les activités, les rôles et les acteurs selon le scénario de reconfiguration choisi.

Après avoir analysé les processus à travers les concepts d'activité, de rôle et d'acteur, nous allons évaluer le choix de(s) scénario(s) de reconfiguration selon des critères qui permettent de juger l'efficacité du scénario afin de réussir la phase de reconfiguration.

L'étape suivante consiste à mettre en œuvre les actions de reconfiguration déterminées. Enfin, la dernière étape est l'évaluation des actions de reconfiguration selon les caractéristiques de reconfiguration (modularité, intégrabilité, convertibilité, diagnosticabilité et personnalisation).

Notre approche illustrée sur la figure 1 suggère un ensemble d'étapes permettant d'aboutir à la proposition d'un ensemble d'actions de reconfiguration au niveau des processus métiers de la chaîne logistique. 


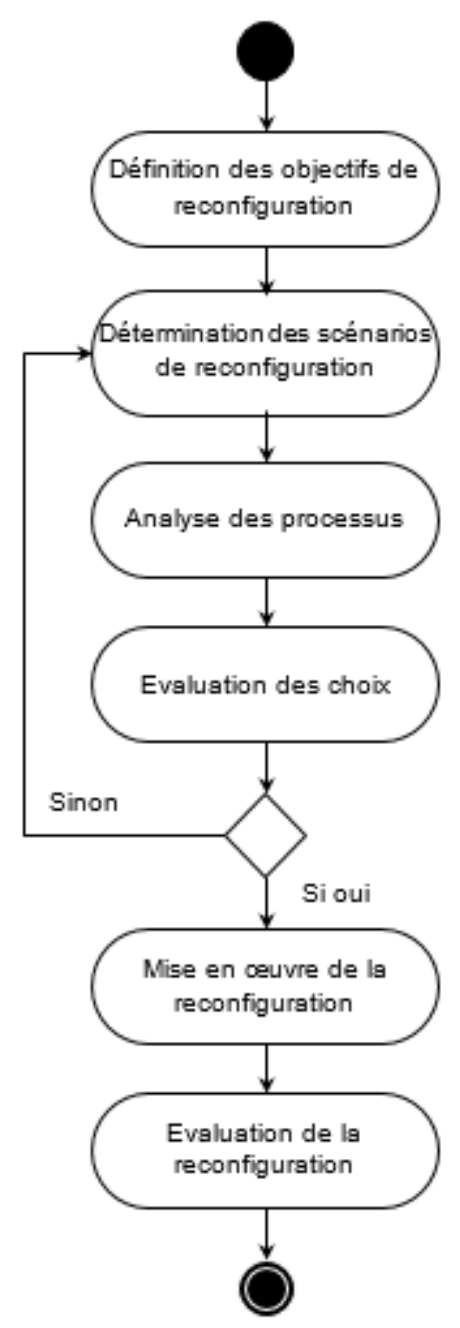

Figure 1. Diagramme d'activité de la démarche de reconfiguration

Pour ce faire, nous nous basons sur les travaux de Mouawad (2010) et Kermad (2018) qui ont proposé des approches de conduite du changement dans les projets d'intégration. Mouawad (2010) s'est focalisé sur la mise en place de l'ERP dans une entreprise industrielle intégrée, ce qui fait que les objectifs de performance sont exprimés en fonction des processus internes. Par la suite, Kermad (2018) s'est intéressé aux objectifs de performances qui sont exprimés en fonction des processus externes dans le cadre de l'entreprise virtuelle (entreprise étendue). Il a proposé une méthodologie composée de quatre étapes qui consiste à modéliser le réseau d'entreprises et les motivations stratégiques des différents partenaires, ensuite recueillir et modéliser des situations d'interopérabilité des processus métiers et des scénarios d'échange qui leur sont associés, puis spécifier les chaînes de services applicatifs qui prennent en charge ces scénarios métiers et enfin définir le modèle d'exécution qui sera simulé afin de préparer la bonne configuration.

Kermad (2018) s'est appuyé sur les différentes phases proposées par Mouawad (2010) tout en gardant la première et la troisième phase et en remplaçant la phase de détermination des caractéristiques organisationnelles par une phase de caractérisation et d'hiérarchisation des rôles et d'une phase de détermination des caractéristiques des acteurs.

\subsection{Définition des objectifs de reconfiguration}

Le besoin de la reconfiguration est déclenché suite à un aléa (dysfonctionnement...) au niveau de la chaîne logistique. Cette défaillance affecte, entre autres, les processus métiers de la chaîne, c'est pour cette raison que nous allons nous baser sur les notions d'activité, rôle et acteur pour pouvoir mettre en place les bonnes pratiques pour la reconfiguration des processus métiers de la chaîne logistique. 
L'identification des objectifs consiste essentiellement à délimiter le problème de reconfiguration pour se focaliser sur les processus (les activités, les rôles et les acteurs) concernés.

\subsection{Détermination des scénarios de reconfiguration}

Notre approche consiste à hiérarchiser les scénarios de reconfiguration en tenant compte des différents objectifs fixés auparavant pour déterminer le scénario le plus approprié. Le terme «scénario » a été défini par Morley et al. (2011) comme étant une description de la simulation d'une instance de processus détaillé, qui permet d'illustrer les nouvelles options pour un nouveau processus. Pour mettre en œuvre cette étape, nous allons nous baser sur la méthode d'Analyse Hiérarchique des Procédés (AHP) développée par Saaty (1990). C'est une méthode multicritère d'aide à la décision permettant aux preneurs de décision d'établir des priorités pour les différentes alternatives afin d'aboutir à un choix de la meilleure décision.

En entrée de la méthode AHP, les décideurs génèrent à chaque niveau de décision un poids pour les différents critères d'évaluation selon des matrices de comparaison. En sortie de la méthode, le classement préférentiel est déterminé par la normalisation du vecteur propre des matrices de comparaison qui correspond aux poids des critères.
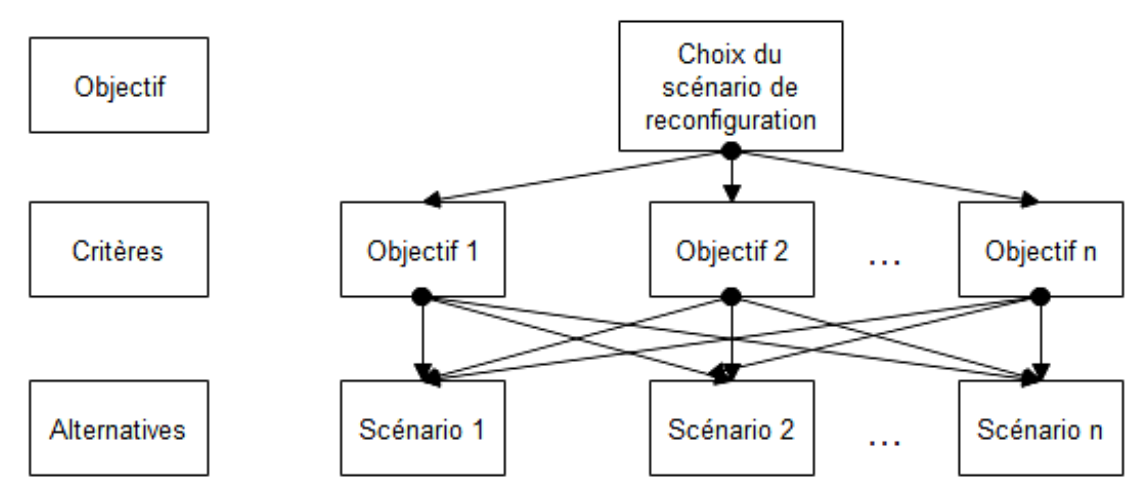

Figure 2. Structure hiérarchique du problème de reconfiguration

Les preneurs de décision établissent une matrice de comparaison par paire appelée $A$ entre les différents critères (les objectifs de reconfiguration). Cette matrice est composée des éléments $a_{i j}^{0}$ qui désignent la valeur d'importance indiquant la comparaison entre l'élément $i$ et l'élément $j$.

$$
A=\left[a_{i j}^{0}\right]=\left(\begin{array}{cccc}
1 & a_{12}^{0} & \cdots & a_{1 n}^{0} \\
\frac{1}{a_{12}^{0}} & 1 & \cdots & a_{2 n}^{0} \\
\vdots & \vdots & \ddots & \vdots \\
\frac{1}{a_{1 n}^{0}} & \frac{1}{a_{2 n}^{0}} & \cdots & 1
\end{array}\right)
$$

La détermination du scénario de reconfiguration à choisir nécessite l'agrégation de tous les poids obtenus dans la matrice (scénarios, objectifs) illustrée dans le tableau 2. 


\begin{tabular}{c|ccc|c} 
& Obj $_{1}$ & $\ldots$ & $\mathbf{O b j}_{\mathbf{n}}$ & $\mathbf{w}_{\mathbf{k}}$ \\
\hline $\boldsymbol{w}_{\boldsymbol{k}}^{\mathbf{1}}$ & $w_{1}^{1}$ & $\ldots$ & $w_{\mathrm{n}}^{1}$ & \\
\hline $\mathbf{S c}_{\mathbf{1}}$ & $w_{11}^{2}$ & $\ldots$ & $w_{1 \mathrm{n}}^{2}$ & $\mathrm{~W}_{1}$ \\
$\vdots$ & $\vdots$ & $\ldots$ & $\vdots$ & $\ldots$ \\
$\mathbf{S c}_{\mathbf{n}}$ & $w_{n 1}^{2}$ & $\ldots$ & $w_{n n}^{2}$ & $\mathrm{Wn}_{\mathrm{n}}$
\end{tabular}

Tableau 2. Classement préférentiel des objectifs résultant de l'analyse

\subsection{Analyse des processus}

Le scénario de reconfiguration choisi est destiné à être appliqué au niveau des processus métiers de la chaîne logistique. Nous allons décomposer le processus selon ses trois concepts de bases : l'activité, le rôle et l'acteur. Cette étape décrit en premier lieu l'identification de ces derniers et la détermination de leur corrélation et, en deuxième lieu, le meilleur processus de réalisation du scénario de reconfiguration qui réussira à atteindre les objectifs fixés dans la première étape.

La phase d'analyse des processus nous permet de traiter le problème de reconfiguration à travers les trois concepts de base : activité, rôle et acteur. Après avoir identifié les scénarios de reconfiguration possibles, nous étudions la corrélation entre ces derniers et les processus métiers. La reconfiguration des processus métiers consiste à déterminer les corrélations entre :

- Les scénarios et les activités et la hiérarchisation des activités ;

- Les activités et les rôles et la hiérarchisation des rôles ;

- Les rôles et les acteurs et la hiérarchisation des acteurs.

La Figure 3 illustre cette démarche, qui vise à identifier les maisons de qualité, au sens du QFD, relatives aux processus, activités, rôles et acteurs. . 


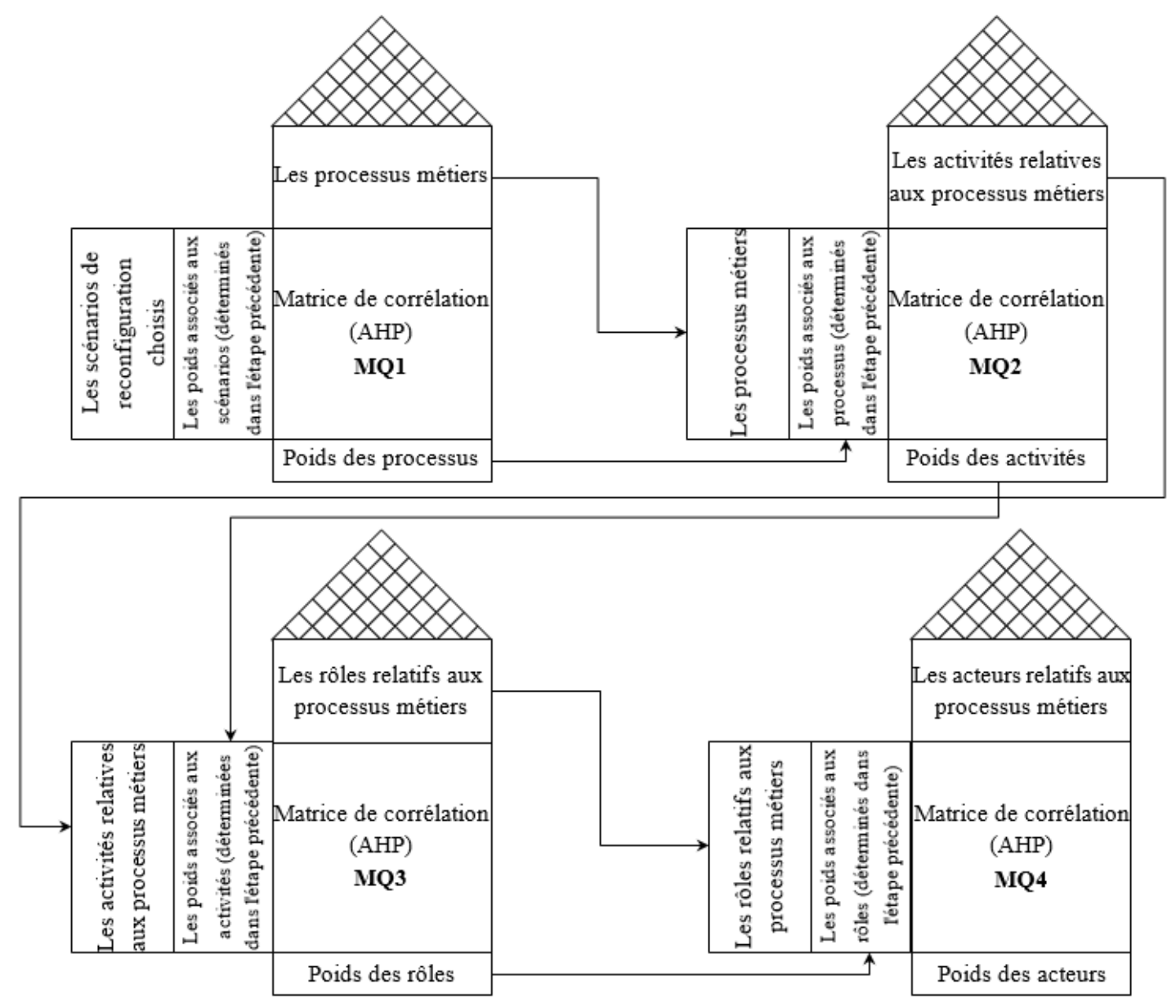

Figure 3. La méthode QFD-AHP appliquée à la reconfiguration des processus métiers de la chaîne logistique

Chaque phase passe par deux étapes principales :

- L'identification du concept (le processus, l'activité, le rôle, l'acteur)

- La hiérarchisation du concept

Nous désignons par $a_{i j}^{1}$ la valeur d'importance qui indique la comparaison des processus de réalisation d'un scénario de reconfiguration selon le critère défini (le scénario de reconfiguration) pour la première maison de qualité. La comparaison sera, ici encore, réalisée par l'analyse proposée par Saaty (1990).

$$
A=\left[a_{i j}^{1}\right]=\left(\begin{array}{cccc}
1 & a_{12}^{1} & \cdots & a_{1 n}^{1} \\
\frac{1}{a_{12}^{1}} & 1 & \cdots & a_{2 n}^{1} \\
\vdots & \vdots & \ddots & \vdots \\
\frac{1}{a_{1 n}^{1}} & \frac{1}{a_{2 n}^{1}} & \cdots & 1
\end{array}\right)
$$

La détermination de la corrélation permet de simplifier l'analyse des comparaisons entre les processus, le compromis de choix et la définition des processus les plus critiques, c'est-à-dire ceux qui doivent être pris en charge en priorité. Certains processus sont indépendants les uns des autres. Ainsi, cette étape permet d'optimiser les processus, par exemple à travers ceux qui n'apportent pas de valeur ajoutée ou ceux qui se répètent. Le calcul du poids se base essentiellement sur les valeurs des degrés de corrélation et le poids de chaque scénario $\mathrm{k}$ obtenu dans la première étape. Par ailleurs, le poids de chaque processus i noté $p_{i}$ est égal à : $p_{i}=\sum_{k=1}^{n} b_{i k} w_{k}$ (Figure 4) 


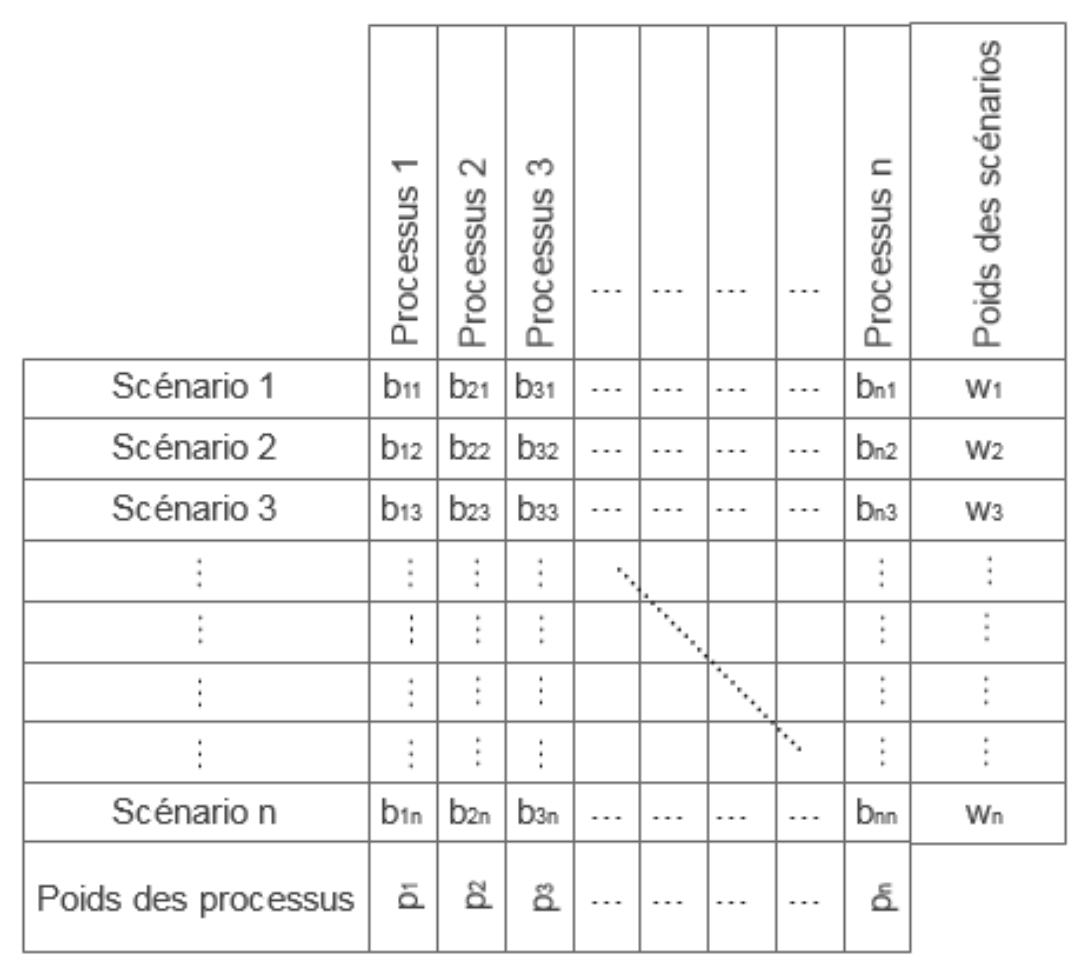

Figure 4. Classement préférentiel des processus résultant de l'analyse

\subsection{Evaluation des choix}

Le scénario de reconfiguration choisi doit être évalué par rapport à un ensemble de critères que nous avons fixés selon les caractéristiques de reconfiguration des chaînes logistiques mentionnées dans la littérature. Nous allons nous baser sur ce qui a été proposé par Biswas (2017) pour déterminer les différents facteurs relatifs à chaque caractéristique d'une chaîne reconfigurable (Tableau 3). Ces facteurs permettent de faciliter le changement dans la chaîne logistique et d'atteindre la satisfaction des caractéristiques.

\begin{tabular}{ll}
\multicolumn{1}{c}{ Caractéristiques } & \multicolumn{1}{c}{ Facteurs } \\
\hline Modularité & La conception du réseau, la réactivité, le temps de cycle total \\
Intégrabilité & L'information en temps réel, la collaboration, la réduction du prix du transfert \\
Convertibilité & L'adaptabilité, la leagility, la confiance \\
Diagnosticabilité & La visibilité, la fiabilité des données, la résilience \\
Personnalisation & La satisfaction du client, la durabilité
\end{tabular}

Tableau 3. Caractéristiques d'une chaîne logistique reconfigurable Biswas (2017)

L'évaluation des scénarios de reconfiguration sera effectuée par une matrice QFD qui permet de déterminer le degré de satisfaction des critères noté $q_{n}$. Cette étape permet de déterminer la corrélation entre le(s) scénario(s) de reconfiguration choisi(s) et les critères d'évaluation notés $f_{i j}$. En effet, le poids relatif à chaque critère montre le degré de satisfaction de ce dernier. Si le scénario de reconfiguration ne satisfait pas les critères mentionnés, le décideur devrait revenir à la deuxième étape pour déterminer le scénario de reconfiguration qui pourra satisfaire ces critères.

\section{Exemple d'illustration}

Pour l'application de notre approche, nous nous basons sur l'exemple proposé par Bellâaj et al. (2011). Ces auteurs ont proposé une démarche d'adaptation aux risques liés à différentes stratégies d'externalisation afin de déterminer la meilleure solution pour le choix de la stratégie de 
l'externalisation. Nous allons appliquer notre approche pour déterminer le processus métier nécessité par la reconfiguration d'une stratégie d'externalisation.

Notre approche est appliquée dans le cas de la SOciété de Production des Articles en Laiton (SOPAL) qui est leader en Tunisie dans la fabrication et la distribution des produits et des équipements sanitaires, du branchement d'eau et du branchement gaz.

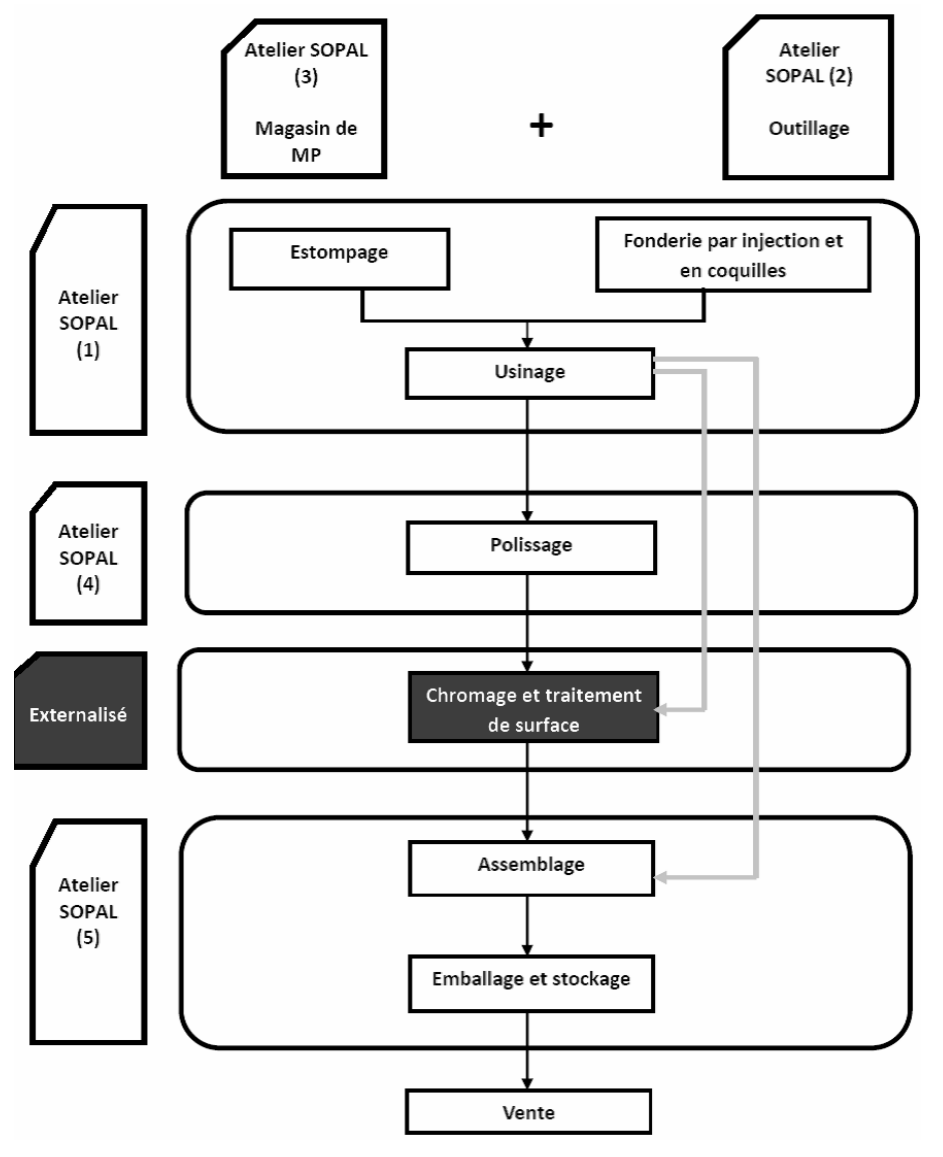

Figure 5. Logigramme de production de SOPAL Bellâaj et al. (2011)

SOPAL maîtrise les procédés de production suivants comme l'illustre la figure 5 :

- Estompage à chaud ;

- La fonderie par injection et en coquilles ;

- L'usinage à commande numérique ;

- Le polissage.

Pour cette application, nous avons construit trois niveaux hiérarchiques :

- Niveau $n^{\circ} 1$ (objectif) : choix du scénario de reconfiguration ;

- Niveau $\mathrm{n}^{\circ} 2$ (critères) : Satisfaction du client, réduire le temps, garantir une information fiable en temps réel, réduire le coût ;

- Niveau ${ }^{\circ} 3$ (alternatives) : Internalisation et externalisation. 


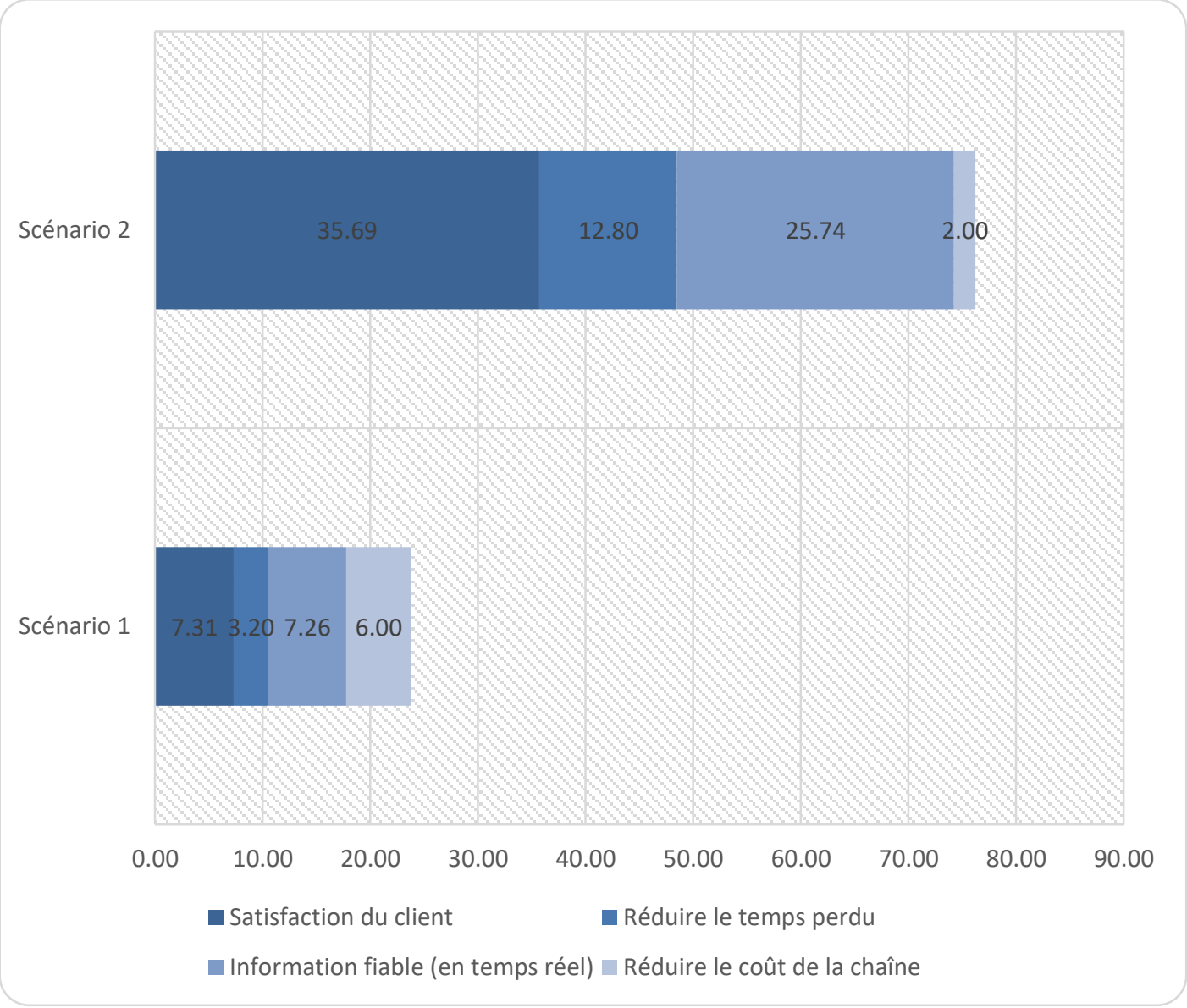

Figure 6. Hiérarchisation des scénarios

Pour le niveau 1, l'analyse AHP nous indique que le scénario 2 (l'externalisation) est celui qui satisfait le plus les objectifs de reconfiguration (Figure 6). Nous concluons que la société a intérêt à externaliser.

Nous menons ensuite une analyse des processus pour déterminer la corrélation entre les scénarios de reconfiguration et le processus métier et de déterminer la corrélation entre ce dernier et les activités, les rôles et les acteurs qui le composent. Nous obtenons ainsi les maisons de qualité des scénarios, des processus, des activités et des rôles (Tableaux 4 à 7).

\begin{tabular}{|c|c|c|}
\cline { 2 - 3 } \multicolumn{1}{c|}{} & Chromage et traitement de surface & Poids des scénarios \\
\hline Scénario 1 & 1 & 0,24 \\
\hline Scénario 2 & 1 & 0,76 \\
\hline Poids des processus : $\mathrm{p}_{\mathrm{n}}$ & 1 & \multicolumn{1}{|c}{} \\
\hline
\end{tabular}

Tableau 4. Maison de Qualité MQ1

L'identification des activités consiste à décomposer le processus de chromage sous un ensemble d'activités. Cette étape permet de déterminer l'activité qui doit être reconfigurée en priorité.

\begin{tabular}{|c|c|c|c|}
\cline { 2 - 4 } \multicolumn{1}{c|}{} & Rinçage & Séchage & Poids des processus \\
\hline Chromage et traitement de surface & 0,83 & 0,17 & 1 \\
\hline Poids des activités $: v_{n}$ & 0,83 & 0,17 & 17 \\
\hline
\end{tabular}

Tableau 5. Maison de Qualité MQ2 
Par ailleurs, l'identification des rôles consiste à définir les rôles associés au rinçage et séchage.

\begin{tabular}{|c|c|c|c|}
\cline { 2 - 4 } \multicolumn{1}{c|}{} & $\begin{array}{c}\text { Rincer les composantes avec l'eau } \\
\text { distillée }\end{array}$ & Sécher les composantes & Poids des activités \\
\hline Rinçage & 0,83 & 0,17 & 0,83 \\
\hline Séchage & 0,20 & 0,80 & 0,17 \\
\hline Poids des rôles $: x_{n}$ & 0,72 & 0,28 & \\
\hline
\end{tabular}

Tableau 6. Maison de Qualité MQ3

La dernière étape de la phase d'analyse des processus consiste à hiérarchiser les acteurs chromeur 1 et chromeur 2 relatifs au processus de chromage.

\begin{tabular}{|c|c|c|c|}
\hline & Chromeur 1 & Chromeur 2 & Poids des rôles \\
\hline Rincer les composantes avec l'eau distillée & 0,89 & 0,11 & 0,72 \\
\hline Sécher les composantes & 0,86 & 0,14 & 0,28 \\
\hline
\end{tabular}

Tableau 7. Maison de Qualité MQ4

Au final, notre analyse des processus métiers conduit à prioriser les activités, les rôles et les acteurs relatifs au processus de chromage et de traitement de surface :

$-\mathrm{v} 1=0,83>\mathrm{v} 2=0,17$

$-\mathrm{x} 1=0,72>\mathrm{x} 2=0,28$

$-\mathrm{y} 1=0,88>\mathrm{y} 2=0,12$

Ainsi, l'activité de rinçage dévolue au chromeur 1 doit être reconfigurée en priorité.

La dernière étape consiste à évaluer le scénario choisi par rapport à la modularité, la convertibilité, l'intégrabilité, la diagnosticabilité et la personnalisation.

\begin{tabular}{|c|c|c|c|c|c|c|}
\cline { 2 - 6 } \multicolumn{1}{c|}{} & Modularité & Convertibilité & Intégrabilité & Diagnosticabilité & Personnalisation & $\begin{array}{c}\text { Poids } \\
\text { des } \\
\text { scénarios }\end{array}$ \\
\hline Externalisation & 0,4 & 0,1 & 0,1 & 0,25 & 0,15 & 1 \\
\hline Poids des critères $: \mathrm{q}_{\mathrm{n}}$ & 0,4 & 0,1 & 0,1 & 0,25 & 0,15 & \\
\hline
\end{tabular}

Tableau 8. Maison de la Qualité MQ5

Les résultats d'évaluation montrent que l'externalisation du processus de chromage satisfait notamment le critère de modularité avec un poids $\mathrm{q}_{1}=0,4$, par la minimisation du temps de cycle et la minimisation du coût global de la chaîne logistique. La diagnosticabilité possède un poids $\mathrm{q}_{4}=0,25$ à travers l'assurance de la fiabilité des informations. La personnalisation possède un poids $\mathrm{q}_{5}=0,15$ en garantissant la satisfaction du client. Enfin, la convertibilité et l'intégrabilité possèdent la même valeur du poids $\mathrm{q}_{2}=\mathrm{q}_{3}=0,1$.

Pour conclure sur ce cas d'application, le scénario d'externalisation permet de répondre aux objectifs de reconfiguration définis dans la première étape. Notre approche nous a permis de déterminer l'activité, le rôle et l'acteur devant prioritairement être reconfigurés en rapport avec le besoin d'externalisation. 


\section{Conclusion}

Dans cet article, nous avons abordé la problématique de la reconfiguration des processus métiers dans les chaînes logistiques en introduisant les concepts d'activité, de rôle et d'acteur. Cette décomposition nous a permis d'optimiser la reconfiguration en hiérarchisant les processus selon ces concepts et en s'appuyant sur l'hybridation des approches QFD/AHP.

En perspective, nous proposons d'améliorer notre approche par une phase d'évaluation de la reconfiguration après sa mise en œuvre. Enfin, nous envisageons de prendre en compte l'incertitude sur les informations disponibles au moment de prendre une décision, en exploitant le potentiel de la méthode AHP vis-à-vis de l'incertitude des données.

\section{Bibliographie}

Bellâaj S., El Mhamedi A., Boujelbène Y., and Kermad L., (2011), « Adaptation to the risks related to outsourcing strategy », International Journal of Business Continuity and Risk Management, Vol. 2, No. 1, pp.35-41.

Biswas P., (2017), « Modeling reconfigurability in supply chains using total interpretive structural modeling », Journal of Advances in Management Research, Vol. 14, No. 2, pp.194-221.

Chandra C., Grabis J., (2016), Supply Chain Configuration, Springer, 1-297.

Chandra C., Kamrani A., (2004), Mass customization : a supply chain approach, Springer Science \& Business Media.

Chandra C., Tumanyan A., (2002), «Supply Chain Reconfiguration : Designing Information Support with System Taxonomy Principles », Proceedings : Eleventh Annual Industrial Engineering Research Conference (IERC), May 1922, Orlando, Florida.

Changchien S., Hsiao-Yun S., (2002), « Supply chain reengineering using a core process analysis matrix and objectoriented simulation », Information \& Management, Vol. 39, No. 5, pp. 345-358.

Charnes A., Cooper W. W., Rhodes E. L., (1978), « Measuring the efficiency of decision making units », European Journal of Operational Research, 2(6), pp. 429-444.

Dev k., Shankar R., Dey P., (2014), « Reconfiguration of supply chain network : an ISM-based roadmap to performance », Benchmarking : An International Journal, Vol. 21, No. 3, pp. 386-411.

Dev k., Shankar R., Gunasekaran A., Thakur L., (2016), " A hybrid adaptive decision system for supply chain reconfiguration », International journal of production research, Vol. 54, No. 23, pp. 7100-7114.

Hvolby H., Trienekens J., (2001), « Models for Supply Chain Reengineering », Production Planning and Control, Vol. 12, No. 3, pp. 254-264.

Ivanov D., Sokolov B., Kaeschel., (2009), « Structure dynamics control-based framework for adaptive reconfiguration of collaborative enterprise networks », International Journal of Manufacturing Technology and Management, Vol. 17, No.1, pp. 23-41.

Ivanov D., Sokolov B., and Kaeschel J., (2010), « A multi-structural framework for adaptive supply chain planning and operations control with structure dynamics considerations », European journal of operational research, Vol. 200, No. 2, pp. 409-420.

Kelepouris T., Wong C., Farid A., McFarlane D., (2006), «Towards a Reconfigurable Supply Network Model », Intelligent Production Machines and Systems 2nd I*PROMS Virtual International Conference, 3-14 July 2006.

Kermad L., (2018), Contribution à la conduite du changement dans les projets d'intégration organisationnelle des entreprises, Mémoire de HDR, Université Paris 8

Koren Y., Heisel U., Jovane F., Moriwaki T., Pritschow G., Ulsoy G., Van Brussel H., (1999), « Reconfigurable manufacturing systems », CIRP Ann Manuf Technol, Vol. 48, pp. 527-540

Mashari M., Mohamed Z., (2000), « Revisiting BPR : a holistic review of practice and development », Business Process Management Journal, Vol. 6, No. 1, pp. 10-42

Morley C., Figueiredo M., Gilette Y., (2011), Processus métiers et SI-3e éd. : Gouvernance, management, modélisation, Dunod.

Mouawad P., (2010), Contribution à l'intégration des méthodes de gestion de la qualité dans les projets d'implantation des ERP, Thèse de Doctorat, Université Paris 8. 
Palma-Mendoza JA., Neailey k., Roy R., (2014), « Business process re-design methodology to support supply chain integration », International Journal of Information Management, Vol. 34, No. 2, pp. 167-176.

Ross A., Venkataramanan M., W.Ernstberger K., (1998), « Reconfiguring the supply network using current performance data », Decision Sciences, Vol. 29, No. 3, pp. 707-728.

Saaty T. L., (1990), « How to make a decision : the analytic hierarchy process », European journal of operational research, Vol. 48, No. 1, pp. 9-26.

Masood T., Weston R., Rahimifard A., (2013), «A model-driven approach to enabling change capability in SMEs », The International Journal of Advanced Manufacturing Technology, Vol. 69, pp. 805-821.

Thierry C., Thomas A., Bel G., (2008), La simulation pour la gestion des chaînes logistiques, Hermès Science Publications-Lavoisier.

Vernadat F., (1992), CIM-OSA -A European development for enterprise integration, Part 2 : Enterprise modelling. C. Petrie (Ed.), Enterprise Integration Modelling, MIT Press, Cambridge, MA (1992), pp. 189-204. 